\title{
International survey of veterinarians to assess the importance of competencies in professional practice and education
}

\begin{abstract}
Objectived To determine the perceived importance of specific competencies in professional veterinary practice and education among veterinarians in several countries.

Designð Survey-based prospective study.

Sampleð 1,137 veterinarians in 10 countries.

Proceduresठ Veterinarians were invited via email to participate in the study. A framework of 18 competencies grouped into 7 domains (veterinary expertise, communication, collaboration, entrepreneurship, health and welfare, scholarship, and personal development) was used. Respondents rated the importance of each competency for veterinary professional practice and for veterinary education by use of a 9-point Likert scale in an online questionnaire. Quantitative statistical analyses were performed to assess the data.

Resultsठ All described competencies were perceived as having importance (with overall mean ratings [all countries] Ó 6.45/9) for professional practice and education. Competencies related to veterinary expertise had the highest ratings (overall mean, 8.33/9 for both professional practice and education). For the veterinary expertise, entrepreneurship, and scholarship domains, substantial differences (determined on the basis of statistical significance and effect size) were found in importance ratings among veterinarians in different countries.
\end{abstract}

Conclusions and Clinical Relevanceð Results indicated a general consensus regarding the importance of specific types of competencies in veterinary professional practice and education. Further research into the definition of competencies essential for veterinary professionals is needed to help inform an international dialogue on the subject.

Keyword: Professional competence; Veterinarians; Education 